\title{
How to provide continuing care during the COVID-19 pandemic: 1-month experience from the Neurosurgery Department in the French University Hospital Timone in Marseille
}

\author{
Nathan Beucler ${ }^{1} \wedge$, Anis Choucha ${ }^{1}$, Sébastien Boissonneau ${ }^{1}$, Thomas Graillon $^{1,2}$, Mikael Meyer $^{1}$, \\ Kaissar Farah ${ }^{1}$, Faisal Albader ${ }^{1}$, Lionel Velly ${ }^{3}$, Pierre-Edouard Fournier ${ }^{4}$, Romain Carron ${ }^{5}$, \\ Stéphane Fuentes ${ }^{1}$, Henry Dufour ${ }^{1,6}$
}

\begin{abstract}
${ }^{1}$ Neurosurgery Department, APHM, Timone University Hospital, Marseille, France; ${ }^{2}$ Aix-Marseille Université, CNRS, CRN2M, UMR 7286, Marseille, France; ${ }^{3}$ Intensive Care Unit, APHM, Timone University Hospital, Marseille, France; ${ }^{4}$ Infectious Diseases Department, HospitaloUniversity Institute Méditerranée Infection, Timone University Hospital, Marseille, France; ${ }^{5}$ Stereotactic and Functional Neurosurgery Department, APHM, Timone University Hospital, Marseille, France; 'Aix-Marseille University, INSERM, MMG, Marseille, France

Contributions: (I) Conception and design: N Beucler, H Dufour; (II) Administrative support: M Meyer, L Velly, PE Fournier, S Fuentes, H Dufour; (III) Provision of study materials or patients: All authors; (IV) Collection and assembly of data: N Beucler, A Choucha, M Meyer, S Fuentes, H Dufour; (V) Data analysis and interpretation: All authors; (VI) Manuscript writing: All authors; (VII) Final approval of manuscript: All authors.

Correspondence to: Nathan Beucler, MD. Neurosurgery Department, APHM, Timone University Hospital, 264 rue Saint-Pierre, 13005, Marseille, France. Email: nathan.beucler@neurochirurgie.fr.
\end{abstract}

\begin{abstract}
Background: At the beginning of the COVID-19 pandemic in 2020, the hospital system struggled with the sudden high influx of patients infected by the SARS-CoV-2. As neurosurgeons, we had to maintain a high standard of care for urgent neurosurgical patients with limited resources and sometimes limited access to intensive care unit (ICU) beds. In the same time, we also had to keep the patients and the staff safe from viral contamination. The purpose of this study was to describe the reorganization and the activity of the Neurosurgery Department of the French University Hospital Timone during the first month of national confinement, compared to the same period last year.
\end{abstract}

Methods: We performed a one-month retrospective observational study, and a literature review focused on neurosurgery in the context of COVID-19 pandemic.

Results: We introduced strict isolation measures, and we only performed surgery for urgent patients. Thus, we were able to operate on every patient within the same delay compared to last year (4.2 vs. 3.6 days). Our activity dropped moderately to $62 \%$ of usual. What is more, no patient and nobody from the staff suffered from viral contamination.

Conclusions: In this setting of worldwide viral pandemic, the compliance with international recommendations made it possible to operate safely on urgent neurosurgical patients.

Keywords: Neurosurgery; neurosurgery department; COVID-19; COVID-19 pandemic

Received: 18 July 2020; Accepted: 29 January 2021; Published: 25 June 2021.

doi: 10.21037/jhmhp-20-107

View this article at: http://dx.doi.org/10.21037/jhmhp-20-107

^ ORCID: 0000-0003-3047-4234. 


\section{Introduction}

Since its first notification as a new type of pneumonia in late November 2019, COVID-19 has evolved to a worldwide outbreak in March 2020. This viral disease caused by SARSCoV-2 (1) is extremely contagious and carries a high casefatality rate, ranging from $0.5 \%$ to $5 \%(2,3)$. The elderly and notably those with comorbidities such as obesity, diabetes, cardiovascular disease, and hypertension, are particularly prone to develop a severe COVID-19-related pneumonia (4). The hospital system was restructured in order to face the sudden high influx of patients. In the same time, the urgent non-COVID-19 patients had still to be managed but also shielded from the exposure to the virus. In this setting, our neurosurgery department had to adapt its daily practice in order to maintain a high standard of care for urgent neurosurgical patients, and to protect the staff and units from viral contamination. Herein we report the organizational alterations implemented in our department during the first month of national confinement in France.

Under these difficult circumstances, we aimed at three main goals:

* To enable patients who could not wait the end of the outbreak to be operated on;

* To avoid any contamination of our COVID-19 negative patients;

* And to protect our staff from viral contamination.

We present the following article in accordance with the MDAR reporting checklist (available at http://dx.doi. org/10.21037/jhmhp-20-107).

\section{Methods}

\section{Study design}

We performed a one-month retrospective observational study focused on the management of urgent neurosurgical patients during COVID-19 outbreak. On the way human and material resources were redistributed in this setting. We compared the daily baseline volume of activity of Marseille, Timone university hospital neurosurgical department with the volume of activity during the first month of national confinement in France which started off on Tuesday, March 17, 2020. We strived to cover every aspect of our department. The following variables or data were analysed or collected:

* Patient recruitment;

* Operating theatre constraints;
* Unit accommodations;

* Department reorganization;

* Human resources re-allocation.

We firstly assessed the amount of reduction of the surgical activity, which determined the needs for medical staff, paramedical staff, and sets the number of hospitalization beds required. We compared our surgical activity from Tuesday, March 17, 2020 to Wednesday, April 15, 2020, to our activity between Tuesday, March 19, 2019 and Wednesday, April 17, 2019.

\section{Database research}

We conducted a literature review on NCBI PubMed database (https://www.ncbi.nlm.nih.gov/pubmed/) with the search terms "Neurosurgery" and "Covid-19". On Sunday, April 19, 2020, the research yielded 54 articles: 30 works were excluded because not related to neurosurgical field. Among the 24 articles related to neurosurgery, we excluded 4 articles that were not relevant regarding the current situation. We only retained 20 articles directly relevant to our topic. There were 17 letter to the editor-like articles, 1 perspective, 1 recommendation and 1 topic review (Figure 1).

In those hard times, readers are looking for authors to share their experience facing the disease. The only thing that mattered is that we were able to operate every patient that needed it, without suffering from the loss of any member of our team.

\section{Statistical analysis}

Categorical variables were presented as numbers and percentages. No statistical analysis was performed for this observational retrospective study.

\section{Ethical approval}

This study was conducted in accordance with the Declaration of Helsinki of 1964 or its further amendments (as revised in 2013). This study was approved by the national Institutional Review Board of the French College of Neurosurgeons registered under number IRB00011687 (January 19th, 2021). Because it is an observational retrospective study, individual consent was waived. All the participants benefited from the highest standard of care available in our institution. 


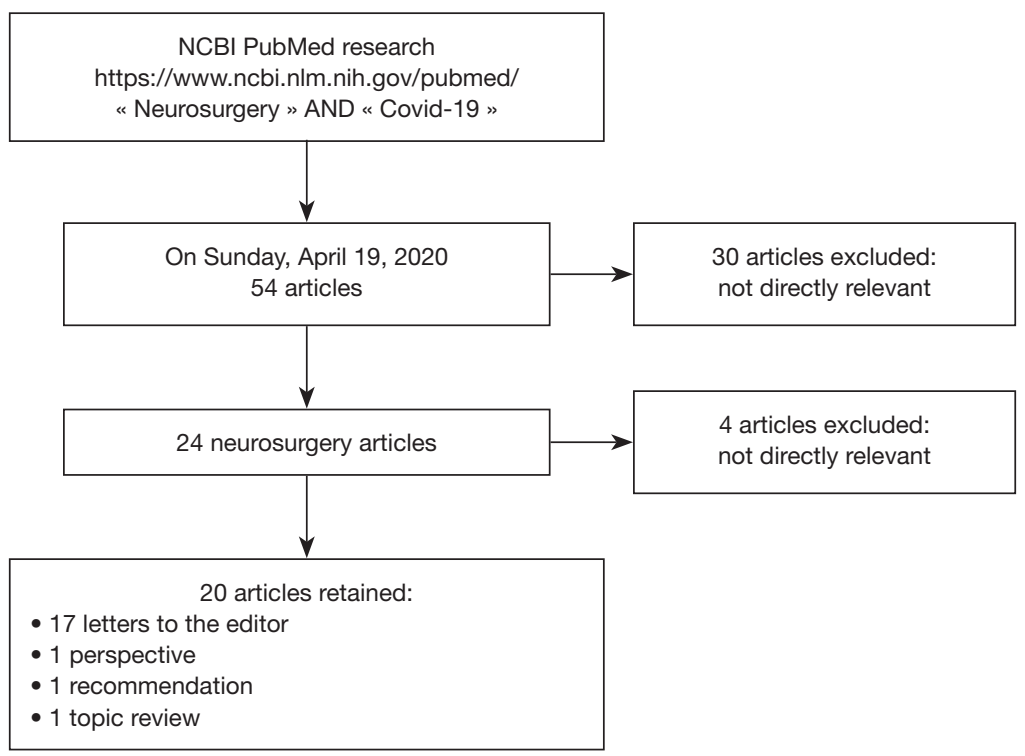

Figure 1 Literature review flowchart.

\section{Results}

\section{General presentation}

Our department is the major neurosurgical center in the district area, acting as a primary center for emergency cases and as a tertiary center for skull base, vascular, oncology and spinal cases. Our team relies on seven senior neurosurgeons and six residents. The department has a total of 61 hospitalization beds, which are divided into one spine unit of 24 beds, and two cranial units of 20 and 17 beds. Last year, we performed 2,400 surgeries, $40 \%$ of them being cranial surgeries and $60 \%$ being spine surgeries. Besides, 10,208 outpatients were seen in consultation.

We have experience working with a team of five interventional neuroradiologists. We benefit from a resuscitation department of 20 beds which is specialized in neurological resuscitation, and an intensive care unit (ICU) of 12 beds. Thus, our facility centralized a great part of neurosurgical emergencies from the entire region during COVID-19 outbreak. However, considering that we were also a big part of regional COVID-19 ICU, we rapidly faced a lack of resuscitation beds for serious neurosurgical patients, as well as for simple postoperative observation after major surgeries.

\section{Patient recruitment}

Neurosurgical patients often face to a worrying diagnosis, and many of them require surgery within a short delay.

As from the announcement of the French emergency "white plan" on Friday, March 13, 2020, all regular outpatient consultations were delayed or cancelled. The patients who were already hospitalized for regular nonurgent surgery were discharged. Thus, we were able to close the unit of 17 beds on Monday, March 23, which freed up space and staff. Because of the reduction of surgical activity, each patient could be installed in an individual room.

From the announcement of national confinement on Tuesday, March 17, 2020, we only accepted patients requiring surgery in a short delay. Upon admission, all patients were classified from 2 (delayable surgery) to 5 (vital emergency) regarding their emergency degree:

2. Surgery within the month (brain tumor, degenerative myelopathy);

3. Surgery within one week (brain tumor/collection with focal deficit, cerebral lymphoma suspicion, surgical site infection, myelopathy/radiculopathy with slight motor deficit, radiculopathy with hyperalgesia);

4. Surgery within 24 hours (any cause of symptomatic intracranial hypertension, aneurysmal subarachnoid haemorrhage, acute hydrocephalus, myelopathy/ radiculopathy with paralysing motor deficit $M R C<3 / 5$ );

5. Surgery immediately required (vital emergency such as acute extra-axial hematoma or hemorrhagic stroke with intracranial hypertension, or functional emergency such as spine trauma with complete/incomplete motor deficit). 
Table 1 Listing of the most common pathologies in neurosurgery

\begin{tabular}{ll}
\hline Procedure & Level \\
\hline Intracranial and/or intraparenchymal hemorrhage regardless of the etiology & 3 to 5 \\
Acute or subacute hydrocephalus & 3 to 5 \\
Intracranial neoplasm with ICH and/or worsening of neurological deficit & 3 to 5 \\
Spinal cord compression, cervical spondylotic myelopathy & 2 to 5 \\
Traumatology (cranial and/or vertebra and/or spinal cord) & 3 to 5 \\
Wound infection, brain abscess, spondylodiscitis & 2 to 5 \\
Malignant neoplasm & 2 to 3 \\
Acute radiculopathy non responding to conservative treatment & 2 to 3 \\
\hline
\end{tabular}

$\mathrm{ICH}$, intracerebral hemorrhage.

A list of the most common pathologies in neurosurgery was established in order to classify the patients from 2 to 5 (Table 1). Level 1 patients, who were mainly diagnosed as outpatients, were eligible for delayable surgery. Level 3 to 5 were considered as urgent patients. A multidisciplinary team with experienced senior neurosurgeons and anaesthetists met every Wednesday to schedule the surgical planning for the upcoming week. At the present time on Monday, April 20, 70 level 1 or 2 patients are waiting for their surgery to be scheduled.

About one-third of the admissions came from urgent consultations, one-third from outside hospitals, and onethird from our emergency department. Upon admission, all patients underwent a complete COVID-19 checkup, including medical history for relevant symptoms (fever, cough, dyspnea, flu-like symptoms, contact with COVID positive patients), infrared temperature-taking and preoperative emergency nasopharyngeal swab testing for SARS-CoV-2 (threshold being defined as positive RT-PCR $<35$ cycles) (5). The result was obtained within 6 hours. A low-dose CT scan of the chest was performed in case of any doubt (negative RT-PCR with clinical signs of pneumonia). They were placed in contact and droplet isolation until PCR result. From 03.17.2020 to 03.25.2020, as we were setting up the COVID-19 organization, nasopharyngeal swabs were not performed routinely: we only had 9/37 (1 positive) patients tested. Nasopharyngeal swabs were performed systematically from the 03.26 .2020 . Since then, sixty-one tests (61 negative) were performed upon admission, six (6 negative) were performed during hospitalization, three (3 negative) had already been performed in another institution, and two were not tested as they were hospitalized in ICU and neurosurgery was just a part of their management. In one month, a total of ninety tests were performed; seventytwo patients were tested once, one patient was tested twice, and four patients were tested four times.

\section{Surgical activity}

Because many health professionals working in the operating theatre were affected by the need of human resources in the COVID-19 ICUs (Table 2), we were forced to close one operating room. We kept opened two operating rooms; one was used for the ongoing surgery, while the other one was ready for any case of level 5 emergency. The anesthesiologist team was reduced from two to one anesthesist and from three to one anesthesia nurse, which was adapted for our new organization to work. Our team of scrub nurses was reduced by half, with 3 nurses at the same time. This helped saving time between surgeries, the third scrub nurse being able to prepare for the next surgery in the other operating room. In order to take advantage of the lack of operative room and optimize operative time, all major procedures were performed "from skin to skin" by experienced senior neurosurgeons. Extended spinal surgeries were performed by a team of two surgeons, operating simultaneously their side of the patients.

Another operating room was also prepared for any case of COVID-19 positive patient. This room was shared with other specialities. The air ventilation in this room was stopped during both steps of intubation and extubation of the patient, in order to minimize the amount of droplets ejected in and out of the room. All necessary material was provided for every health professional to apply strict air and contact isolation (FFP2 masks and protective glasses in addition to usual sterile gloves and surgical gowns). 
Table 2 Neurosurgery department reorganization

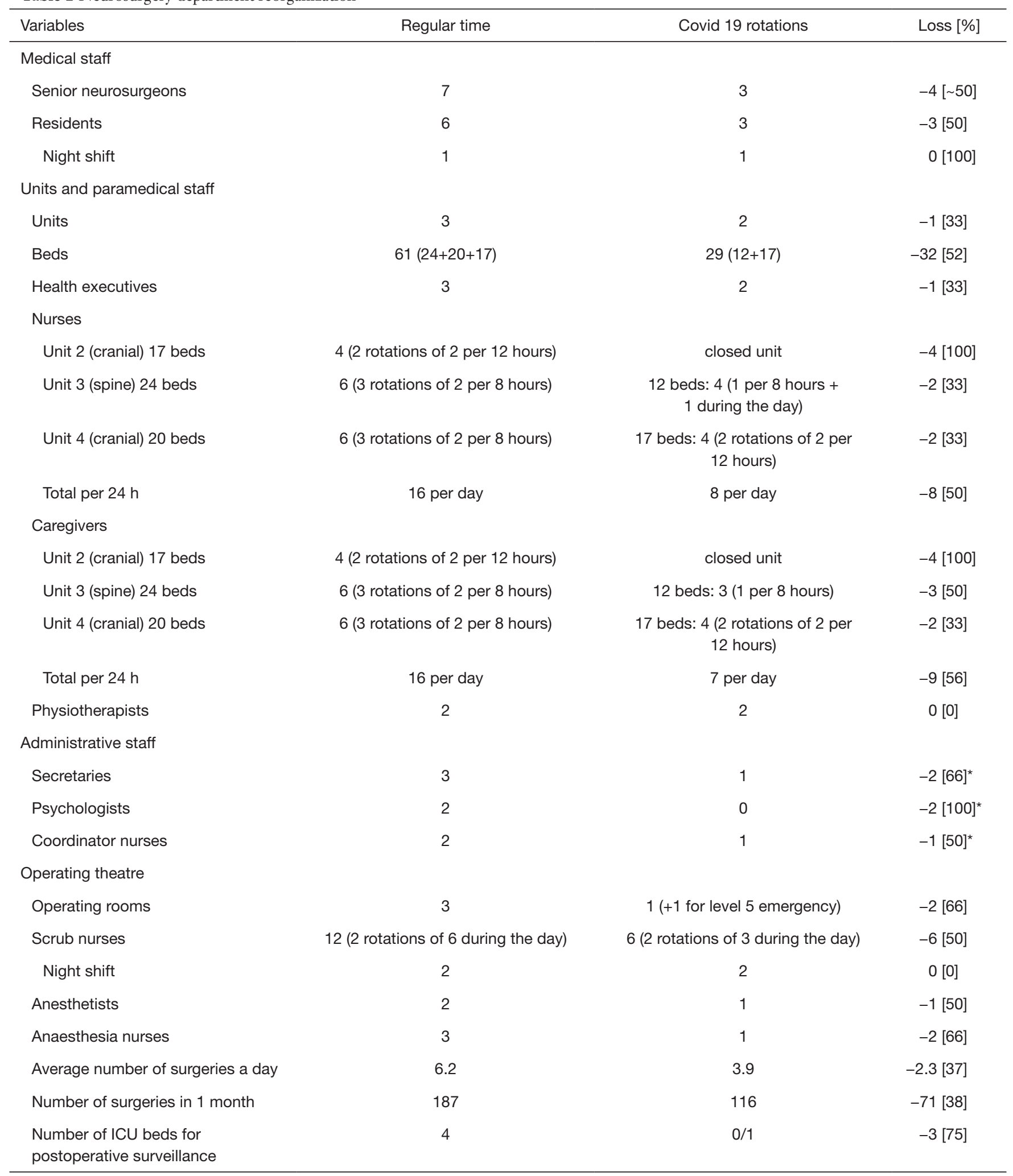

*, teleworking at home. 
Table 3 Comparison between the monthly surgical activity in 2020 and in 2019

\begin{tabular}{lccc}
\hline Level & 2019 & 2020 & Difference \\
\hline Level 5 & $12(6.4 \%)$ & $10(8.6 \%)$ & $-2(16.6 \%)$ \\
Level 4 & $15(8 \%)$ & $18(15.5 \%)$ & $+3(20 \%)$ \\
Level 3 & $77(41 \%)$ & $88(76 \%)$ & $+11(14.3 \%)$ \\
Level 1-2 & $83(44.3 \%)$ & 0 & $-83(100 \%)$ \\
Total & 187 & 116 & $-71(38 \%)$ \\
\hline
\end{tabular}

During the first month of confinement (Tuesday, March 17, 2020 to Wednesday, April 15, 2020), our team performed 116 surgeries, with an average of almost 4 surgeries a day. We performed $88(76 \%)$ level 3 procedures, $18(15.5 \%)$ level 4 procedures, and $10(8.6 \%)$ level 5 procedures. For unscheduled level 3 surgeries, median time for access to surgery was 4.2 days. For level 4 and level 5 patients, priority access was always granted with good results regarding outcome, given the gravity of the patient's initial condition; 56 (48\%) cranial surgeries were performed, among which there were $9(8 \%)$ posterior fossa procedures; 60 (52\%) spine surgeries were carried out.

During the same period last year (Tuesday, March 19, 2019 to Wednesday, April 17, 2019), we performed 187 surgeries, with an average of 6.2 surgeries a day. There were 83 (44\%) level 1 or 2 surgeries, 77 (41\%) level 3 surgeries, 15 (8\%) level 4 surgeries, and 12 (6.4\%) level 5 surgeries. For unscheduled level 3 surgeries, median time for access to surgery was 3.6 days. We almost had the same emergency activity (level 3 to 5 surgeries) during the first month of home confinement compared to last year (number of urgent procedures, $2020 v s$. 2019: level 3, $88 v s$. 77; level 4, $18 v s$. 15; level 5, 10 vs. 12. Plus 11\% difference) (Table 3, Figure 2).

\section{Surgical management of COVID-19 positive patients}

We had to perform three surgeries that carried a worrisome risk of viral transmission. Indeed, three patients were tested COVID-19 positive at the time or around the time of their surgery.

The first one was a 68-year-old patient referred to us by another institution for unstable L4L5 spondylodiscitis. He was admitted in our department before March 16 . He was operated on but the procedure had to be stopped before spinal instrumentation because of hemodynamic trouble. A bed rest with minimal mobilization was advocated and a second operation was planned after cardiac checking and antibiotic therapy. At day 11, he presented symptoms of pneumonia, with a positive nasopharyngeal swab RTPCR and a CT scan of the chest suggestive of COVID-19. He was transferred to COVID-19 medical unit, and was sent back to us after 2 negative SARS-CoV-2 RT-PCR. However, he was kept in contact and droplet isolation for the rest of his hospitalization, and benefited from posterior lumbar osteosynthesis in the COVID-19 operating room.

We also performed a suboccipital decompressive craniectomy in a 54-year-old COVID-19 positive patient, who suffered from malignant cerebellar infarction. This surgery was carried out in the COVID-19 operating room.

These two patients did not present respiratory impairment afterwards, and no clinical event suspect of COVID-19 occurred in the staff present for both operations.

The third one was a 63 -year-old who underwent surgical revision for surgical site infection after posterior lumbar instrumentation. Postoperative day 2, she presented acute respiratory failure from massive inhalation; she was admitted in ICU and her SARS-CoV-2 nasopharyngeal swab was positive. The CT scan of the chest was in favour of inhalation pneumonia. She was successfully extubated and transferred back to our department. All her other SARS-CoV-2 nasopharyngeal swabs were negative. What is more, the RT-PCR result was given "positive" without the usual reference to the number of PCR cycles. Given that this patient did not show clinical or radiologic evidence of infection by the SARS-CoV-2, this "positive" RT-PCR result raises the question of false-positive tests (6).

\section{Particularity of transsphenoidal surgery}

Our colleagues in Wuhan alerted the neurosurgical community regarding the very high risk of contamination of operating room staff during transsphenoidal approach. Hence, we have cancelled all patients who required 

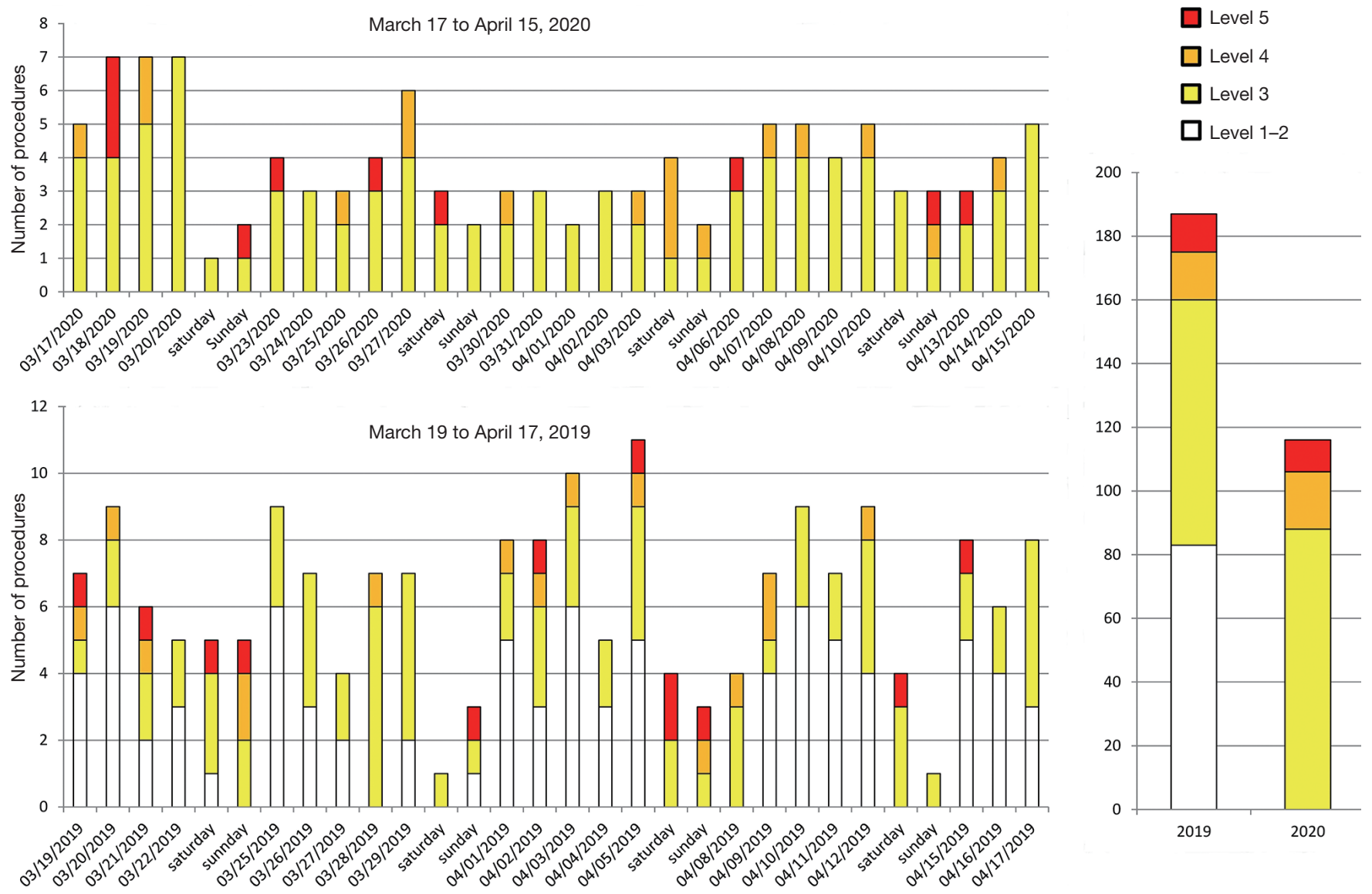

Figure 2 Comparison between the monthly surgical activity in 2020 and in 2019 .

this type of surgery, even those who presented chiasma compression without severe loss of visual acuity. However, we had to perform two emergency debulking surgeries in patients suffering from symptomatic compressive pituitary apoplexy with severe loss of visual acuity, ranked level 4 in our classification system. Only the staff strictly necessary was physically present in the operating room during each stage of the patient's management, and they were provided with the complete personal protective equipment (PPE) required (7-9).

\section{Postoperative management}

One challenge we had to deal with was the difficulty to provide proper postoperative observation for patients who underwent major surgeries. Because of the need to spare places in ICUs for COVID-19 positive patients and the risk to accommodate COVID-19 negative with COVID-19 positive patients, we decided to change our habits for patients with serious pathologies who would have needed at least one night in ICU following the surgery. For example, a few patients who underwent posterior fossa surgery benefited from prolonged observation (6 hours with controlled hypotension) in the recovery room before coming back to their former unit. However, these modalities had been discussed for each patient before. All these patients were seen by both the senior anesthesist and the neurosurgery resident before coming back to the unit, and they benefited from an hour-by-hour surveillance by the unit team all night long. All of the patients treated with these modalities presented an uneventful postoperative course.

Surgical wound closures were performed as usual: some surgeons used dissolvable stitches, some other used surgical staples. Surgical wound care was never an issue because there is an established network of nurses outside the hospital. They were provided with enough PPE to continue to take care of their patients as usual. As a result, we did not 
suffer from additional scar complications.

\section{Unit management and staff protection}

After 17 beds closed, all neurosurgical patients were gathered on a single floor. Every patient was given an individual room and this commitment was strictly fulfilled. In parallel, all visitors were strictly prohibited in the hospital.

Our medical team was reduced to the number necessary to perform the surgeries, look after the patients in the remaining two units, and attend urgent consultations. Half of our senior neurosurgeons stayed at home. Our residents evolved in a rotation planning so that they were only 3 on 6 at the same time in the department. We kept our routine morning staff where we reviewed every case of patient admitted in our department, every postoperative imaging, and every complementary examination.

Beyond patients COVID-19 check-up upon admission, we took every measure to protect our staff and limit physical contact between our team and COVID-19 positive patients. We strived to provide enough surgical masks so that every health professional could have a new one every four hours. Our paramedical staff received 30 minutes individualized training for proper complete air and contact dressing and undressing. They were given FFP2 masks, disposable gowns and gloves for the patient admission process, which comprised systematic nasopharyngeal swab. Every patient who came up with fever, upper or lower respiratory symptoms during hospitalization was given a surgical mask, and a new nasopharyngeal swab was performed to assess for SARS-CoV-2 status. Considering the rate of false-negative tests, a low-dose CT scan of the chest was done in case of any doubt.

We managed to provide physiotherapy care to our patients with focal deficits before surgery and during early postoperative period, and for those who underwent spine surgery, especially after lumbar prolapsed disc surgery. Our two physiotherapists continued to work half-time with us just like they used to, and they were provided PPE during their four hours vacations. Consequently, no patient suffered from loss of chance of neurological recovery.

Mean hospitalization time was 8.4 days for 2020 patients compared to 9.5 days for 2019 patients.

As a matter of facts, with all these measures, no one from our entire neurosurgical team presented any symptom suggestive of COVID-19 infection. Hence, no systematic COVID-19 set-up was organized for our morning medical staff. We did not suffer from patient viral contamination either.

\section{Hospital adaptation to COVID-19 pandemic}

Our institution reorganized into two physically different circuits related to patients SARS-CoV-2 status. The emergency department split into a COVID-19 free unit of 17 beds, and a COVID-19 suspicion unit of 15 beds. All patients with suspicion of COVID-19 or positive nasopharyngeal swab underwent a low-dose CT scan of the chest; one CT scan was specifically assigned to these cases. Our infectious disease department with a capacity of 75 hospitalization beds, and one of the hospital floors became the new COVID-19 medical units with a capacity of 152 beds. These units were provided medical teams with people freed from their former department. We increased our institution resuscitation department capacity from 62 to 126 beds, 78 of them being assigned to COVID-19 positive patients.

Some of our senior neurosurgeons volunteered during their rest time to take care of patients in the COVID-19 medical units. They did 4 hours rotations wearing full-PPE clothing. Besides reminding them of their general medical training, it gave them the feeling to really contribute to the global effort.

\section{University activities and academic teaching}

As from the announcement of the French emergency "white plan" on Friday, March 13, 2020, all university courses were cancelled. However, medical students were given the opportunity to volunteer their services. For example, they have provided nationwide great support in the emergency medical services call centers, and they have been of great assistance for patient stretcher transportation in many hospitals and in the institutions for the elderly. Thanks to the reorganization in our neurosurgery department, we became completely self-sufficient and did not require external assistance.

The French residents usually do 6 months rotations, and they are transferred to a different department every $2^{\text {nd }}$ of November and $2^{\text {nd }}$ of May. Given the seriousness of the situation at the hospital, their rotation was officially postponed until at least the $2^{\text {nd }}$ of June (10). The residents working in less impacted departments could be reassigned to other services under pressure, in accordance to their medical qualifications. Given the amount of neurosurgical 
emergencies, this measure did not apply to the residents in our department.

\section{Discussion}

On Wednesday, March 11, 2020, COVID-19 was spreading worldwide. As the World Health Organization Director Dr. Ghebreyesus declared the pandemic stage of the disease, COVID-19 has already infected 118,000 people in 114 different countries, with a burden of 4,600 direct losses (11). As neurosurgeons, we started to look for help regarding how to continue operating while keeping our team safe, and spare our patients from viral contamination to avoid COVID-19 from becoming a new nosocomial disease as well as a professional pathology. But as we scoured our usual medical literature, it left us unanswered. Thus, we decided that we would give others what we had been looking for. As we write those lines, over 150,000 people are gone because of the COVID-19.

In Italy, where the population has paid a heavy toll to the disease, the designation of reference neurosurgical facilities free of COVID-19 patients was an absolute necessity (12-14). In the south of France, we have done otherwise and have created a completely isolated COVID-19 circuit inside our facility.

Neurosurgery is a small surgical speciality with welltrained physicians (15). Thus, reallocation of neurosurgeons in medical units under pressure had to be thought wisely (16). It depended on the amount of urgent neurosurgical patients and the presence of neurosurgery departments in surrounding hospitals $(12,13)$. Some teams created a rotation planning, so that they could keep their department running and in the same time preserve half of their team (17-19). We followed the same example.

Contact and droplet isolation rules had to be respected in all circumstances $(7-9,20,21)$. The application of these rules implied gradual PPE, from the use of surgical mask in front of every patient, to a complete PPE gear with face and airways protection (N95 or FFP2 mask and protective glasses), surgical gown and gloves during high-risk operations. Clues have also been given for the management of the unit during the pandemic (22), and for the team safety in the $\operatorname{OR}(8,17,23,24)$. A few authors have given a detailed description on how to provide a safe operating room for COVID-19 positive patients $(20,22,25)$. A COVID-19 checklist upon admission was established by many teams $(12,20,22,26-29)$. These checklists agreed on the majority of points that were listed. Taking into account the uncertainty concerning the sensitivity of SARS-CoV-2 nasopharyngeal swab test which is reported up to $60-70 \%$ (30), and the incubation period of the virus, there were more nuances regarding the preoperative observation delay of the patient (31-33). Some authors only asked for a clean medical history and a negative nasopharyngeal swab, while other authors required an observation period of two weeks. We believe that the preoperative checklist and the rigorous compliance with self-protective isolation rules are the keys for a department not to be burdened by the viral infection.

Residents were officially part of the medical task force in many countries $(18,21,24)$. However, taking into account the global reduction of activity in the hospital and for safety reasons, some departments had their residents evolve in rotations.

COVID-19 outbreak led to an unprecedented number of admissions in ICU for severe acute respiratory syndrome in modern medicine era; as a direct consequence, there was a high consumption of medical resources (34). In this setting, neurosurgical teams were obliged to make the difficult choice of prioritizing their patients $(35,36)$. A multidisciplinary COVID-19 team of senior attendings was often created to discuss the emergency level of each patient $(12,26,29)$. A few teams have established simple emergency grading systems, with three different levels of severity $(12,14,22,37)$ : the lowest one was for patients requiring surgery within a week, the medium one for surgery required within the day, and the highest one for immediate vital or functional emergencies. Similar systems have also been introduced specifically for spinal emergencies (38). Finally, some authors simply relied on common sense regarding what is and what is not an emergency (39). All authors agreed that only symptomatic, and vital emergencies should benefit from surgery during the pandemic peak $(19,21,37)$, and a few teams shared their surgical experience according to that principle $(12,23,27,28)$. Any neurosurgical department that continued its emergency activities needed 24 hours in-house medical coverage, which was done by resident night shifts (19). In order to spare operating room occupation time, we believe that doing the right procedure for patients is time saving even if postoperative aftermaths are heavier on the very moment. Among others, Perin et al. and Eichberg et al. raised the same issue we were facing concerning a few major surgeries that could not benefit from postoperative observation in the ICU $(26,28,39)$. Thanks to an inter-hospital organization centralizing COVID-19 positive patients and neurosurgical patients in different facilities, Perin et al. team did not face any 
difficulty for access to postoperative ICU observation. In contrast, our facility was a major center for both neurosurgical emergencies and COVID-19 positive patients. Consequently, our team performed a few major surgeries (for example posterior fossa craniotomies) without postoperative ICU observation.

Authors rapidly discharged their patients; they also developed teleconsultation for postoperative followup, with the use of surgical wound absorbable stitches $(19-21,26)$. In contrast, we were able to absorb the amount of neurosurgical emergencies without changing our habits concerning surgical wounds closure and hospitalization time.

As it could be expected, the activity of the neurosurgical departments still running dropped down to nearly $30 \%$ of usual $(16,26)$. For our part, we faced a smaller reduction in our surgical activity, with a diminution of $38 \%$ compared to the same period last year (Tables 2 and 3, Figure 2).

This activity drop can be explained by numerous factors. First of all, national confinement prevents people from driving or doing dangerous outside activities, which leads to a significant decrease of trauma cases. Moreover, the fearful atmosphere, which is self-sustained by the era of social networks, makes people more likely to stay at home in case of any symptom than to consult in an emergency department (40). Finally, we believe that lots of people are simply too afraid to go to the hospital in a setting of world pandemic. Thus we think that they are more likely to stay at home whatever the seriousness of their condition is, rather than consulting any doctor.

From the beginning of the outbreak in France, and as soon as the nasopharyngeal swabs RT-PCR tests were available, we were rapidly able to test any patient admitted in our department. The Hospital-University Institute Méditerranée Infection has been of great support in order to do so. The detection of asymptomatic positive patients has several advantages, as it avoids intra-hospital contamination for the other patients and the medical staff, and it detects healthy virus carriers during the incubation period.

Lei et al. have reported a series of COVID-19 positive patients operated on during their unknown incubation period, with a mortality rate of $20 \%$ (33). To avoid such an outcome, they recommend a two weeks observation period prior to surgery. This measure is impossible to achieve for level 4 and 5 patients, and difficult for level 3 patients. Taking into account that (I) less than $10 \%$ of the population is infected by the virus, (II) a negative test offers a $70 \%$ chance that the patient is COVID-19 negative, and (III) we have introduced strict barrier measures in the hospital, we have considered that the benefit-risk balance for urgent surgery was reasonable in each case. As a result, only one patient had a respiratory impairment related to COVID-19 infection after the surgery, with a favourable outcome.

\section{Conclusions}

Thanks to a few weeks of preparation, we were able to adapt our organization to the COVID-19 positive patient wave. We increased our institution beds capacity, we reorganized the resuscitation department, and we performed only emergency procedures.

We only wish to share our management choices and procedures, as they were made in accordance with our institution recommendations and needs, and they were supported by recent literature shared by other teams around the world. With this organization, we managed to perform almost 4 surgeries a day, mean time for access to the procedure being 4 days. All the vital and functional emergencies benefited from immediate neurosurgical procedures, and from postoperative ICU COVID-19 free observation bed when absolutely required.

We still don't know how we are going to face the high number of level 1 and 2 patients waiting for their postponed surgery. A few of them are going to become level 3 patients, and are going to benefit from surgery in this context. With the organization described above, at the present time, nobody in our neurosurgical staff developed any sign of infection by the SARS-CoV-2. Additionally, no hospitalized patient requiring neurosurgery has ever experienced a loss of chance due to the COVID-19 outbreak.

\section{Acknowledgments}

Funding: None.

\section{Footnote}

Reporting Checklist: The authors have completed the MDAR reporting checklist. Available at http://dx.doi.org/10.21037/ jhmhp-20-107

Data Sharing Statement: Available at http://dx.doi. org/10.21037/jhmhp-20-107

Peer Review File: Available at http://dx.doi.org/10.21037/ 
jhmhp-20-107

Conflicts of Interest: All authors have completed the ICMJE uniform disclosure form (available at http://dx.doi. org/10.21037/jhmhp-20-107). The authors have no conflicts of interest to declare.

Ethical Statement: The authors are accountable for all aspects of the work in ensuring that questions related to the accuracy or integrity of any part of the work are appropriately investigated and resolved. This study was conducted in accordance with the Declaration of Helsinki of 1964 or its further amendments (as revised in 2013). This study was approved by the national Institutional Review Board of the French College of Neurosurgeons registered under number IRB00011687 (January 19th, 2021). Because it is an observational retrospective study, individual consent was waived.

Open Access Statement: This is an Open Access article distributed in accordance with the Creative Commons Attribution-NonCommercial-NoDerivs 4.0 International License (CC BY-NC-ND 4.0), which permits the noncommercial replication and distribution of the article with the strict proviso that no changes or edits are made and the original work is properly cited (including links to both the formal publication through the relevant DOI and the license). See: https://creativecommons.org/licenses/by-nc-nd/4.0/.

\section{References}

1. Gorbalenya AE, Baker SC, Baric RS, et al. Severe acute respiratory syndrome-related coronavirus: The species and its viruses - a statement of the Coronavirus Study Group. Nat Microbiol 2020;5:536-44.

2. Baud D, Qi X, Nielsen-Saines K, et al. Real estimates of mortality following COVID-19 infection. Lancet Infect Dis 2020;20:773

3. Niforatos JD, Melnick ER, Faust JS. Covid-19 fatality is likely overestimated. BMJ 2020;20;368:m1113.

4. Wu C, Chen X, Cai Y, et al. Risk Factors Associated With Acute Respiratory Distress Syndrome and Death in Patients With Coronavirus Disease 2019 Pneumonia in Wuhan, China. JAMA Intern Med 2020;180:934-43.

5. Gautret P, Lagier JC, Parola P, et al. Hydroxychloroquine and azithromycin as a treatment of COVID-19: results of an open-label non-randomized clinical trial. Int J Antimicrob Agents 2020;56:105949.
6. Tahamtan A, Ardebili A. Real-time RT-PCR in COVID-19 detection: issues affecting the results. Expert Rev Mol Diagn 2020;20:453-4.

7. Lo YT, Yang Teo NW, Ang BT. Editorial. Endonasal neurosurgery during the COVID-19 pandemic: the Singapore perspective. J Neurosurg 2020. doi: 10.3171/2020.4.JNS201036.

8. Patel ZM, Fernandez-Miranda J, Hwang PH, et al. Letter: Precautions for Endoscopic Transnasal Skull Base Surgery During the COVID-19 Pandemic. Neurosurgery 2020;87:E66-E67.

9. World Health Organization. Rational use of personal protective equipment (PPE) for coronavirus disease (COVID-19): interim guidance. 2020. Available online: https://www.who.int/publications/i/item/rational-use-ofpersonal-protective-equipment-for-coronavirus-disease(covid-19)-and-considerations-during-severe-shortages

10. Ministère des Solidarités et de la Santé. instruction relative aux aménagements des modalités de formation pour les étudiants en santé. 2020. Available online: Available online: http://dmg.medecine.parisdescartes.fr/instruction-relativeaux-amenagements-des-modalites-de-formation-pour-lesetudiants-en-sante/

11. WHO Director-General's opening remarks at the media briefing on COVID-19 - 11 March 2020. Available online: https://www.who.int/director-general/speeches/detail/ who-director-general-s-opening-remarks-at-the-mediabriefing-on-covid-19---11-march-2020

12. Cenzato M, DiMeco F, Fontanella M, et al. Editorial. Neurosurgery in the storm of COVID-19: suggestions from the Lombardy region, Italy (ex malo bonum). J Neurosurg 2020. doi: 10.3171/2020.3.JNS20960.

13. Bernucci C, Brembilla C, Veiceschi P. Effects of the COVID-19 Outbreak in Northern Italy: Perspectives from the Bergamo Neurosurgery Department. World Neurosurg 2020;137:465-468.e1.

14. Zoia C, Bongetta D, Veiceschi P, et al. Neurosurgery during the COVID-19 pandemic: update from Lombardy, northern Italy. Acta Neurochir (Wien). 2020;162:1221-2.

15. Roche PH, Cuny E, Régis J, et al. Demography of neurosurgery in France in 2018. Current state and a call to educate more young neurosurgeons. Neurochirurgie 2019;65:4-6.

16. Borsa S, Bertani G, Pluderi M, et al. Our darkest hours (being neurosurgeons during the COVID-19 war). Acta Neurochir (Wien) 2020;162:1227-8.

17. Bambakidis NC, Tomei KL. Editorial. Impact of COVID-19 on neurosurgery resident 
training and education. J Neurosurg 2020. doi: 10.3171/2020.3.JNS20965.

18. Choi BD. Editorial. A neurosurgery resident's response to COVID-19: anything but routine. J Neurosurg 2020. doi: 10.3171/2020.4.JNS201028.

19. Burke JF, Chan AK, Mummaneni V, et al. Letter: The Coronavirus Disease 2019 Global Pandemic: A Neurosurgical Treatment Algorithm. Neurosurgery 2020;87:E50-E56.

20. Liu Z, Zhang Y, Wang X, et al. Recommendations for Surgery During the Novel Coronavirus (COVID-19) Epidemic. Indian J Surg 2020. doi: 10.1007/s12262-02002173-3.

21. Carter BS, Chiocca EA. Editorial. COVID-19 and academic neurosurgery. J Neurosurg 2020. doi: 10.3171/2020.4.JNS201013.

22. Tan YT, Wang JW, Zhao K, et al. Preliminary Recommendations for Surgical Practice of Neurosurgery Department in the Central Epidemic Area of 2019 Coronavirus Infection. Curr Med Sci 2020;40:281-4.

23. Sun Y, Mao Y. Editorial. Response to COVID-19 in Chinese neurosurgery and beyond. J Neurosurg 2020. doi: 10.3171/2020.3.JNS20929.

24. Wellons JC, Grant G, Krieger MD, et al. Editorial. Early lessons in the management of COVID-19 for the pediatric neurosurgical community from the leadership of the American Society of Pediatric Neurosurgeons. J Neurosurg Pediatr 2020. doi: 10.3171/2020.3.PEDS20215.

25. Ti LK, Ang LS, Foong TW, et al. What we do when a COVID-19 patient needs an operation: operating room preparation and guidance. Can J Anesth 2020;67:756-8.

26. Eichberg DG, Shah AH, Luther EM, et al. Letter: Academic Neurosurgery Department Response to COVID-19 Pandemic: The University of Miami/ Jackson Memorial Hospital Model. Neurosurgery 2020;87:E63-E65.

27. Hu YJ, Zhang JM, Chen ZP. Experiences of practicing surgical neuro-oncology during the COVID-19 pandemic. J Neurooncol 2020;148:199-200.

28. Perin A, Servadei F, DiMeco F, et al. May we deliver neuro-oncology in difficult times (e.g. COVID-19)? J Neurooncol 2020;148:203-5.

29. Lee ZD, Chyi Yeu DL, Ang BT, et al. Editorial. COVID-19 and its impact on neurosurgery: our early experience in Singapore. J Neurosurg 2020. doi: 10.3171/2020.4.JNS201026.

30. Ai T, Yang Z, Hou H, et al. Correlation of Chest CT and RT-PCR Testing in Coronavirus Disease 2019
(COVID-19) in China: A Report of 1014 Cases. Radiology 2020;296:E32-E40.

31. Kim H, Hong H, Yoon SH. Diagnostic Performance of CT and Reverse Transcriptase-Polymerase Chain Reaction for Coronavirus Disease 2019: A Meta-Analysis. Radiology 2020;296:E145-E155.

32. Guan WJ, Ni Z, Hu Y, et al. Clinical Characteristics of Coronavirus Disease 2019 in China. N Engl J Med 2020;382:1708-20.

33. Lei S, Jiang F, Su W, et al. Clinical characteristics and outcomes of patients undergoing surgeries during the incubation period of COVID-19 infection. EClinicalMedicine 2020;5;21:100331.

34. Emanuel EJ, Persad G, Upshur R, et al. Fair Allocation of Scarce Medical Resources in the Time of Covid-19. N Engl J Med 2020;382:2049-55.

35. COVIDSurg Collaborative. Global guidance for surgical care during the COVID-19 pandemic: Surgical care during the COVID-19 pandemic. Br J Surg 2020;107:1097-103.

36. American College of Surgeons. COVID-19: elective case triage guidelines for surgical care. 2020. Available online: https://www.facs.org/covid-19/clinical-guidance/electivecase

37. Ramakrishna R, Zadeh G, Sheehan JP, et al. Inpatient and outpatient case prioritization for patients with neuro-oncologic disease amid the COVID-19 pandemic: general guidance for neuro-oncology practitioners from the AANS/CNS Tumor Section and Society for NeuroOncology. J Neurooncol 2020;147:525-9.

38. Ghogawala Z, Kurpad S, Falavigna A, et al. Editorial. COVID-19 and spinal surgery. J Neurosurg Spine 2020. doi: 10.3171/2020.4.SPINE20468.

39. Bernstein M. Editorial. Neurosurgical priority setting during a pandemic: COVID-19. J Neurosurg 2020. doi: 10.3171/2020.4.JNS201031.

40. Guo H, Zhou Y, Liu X, et al. The impact of the COVID-19 epidemic on the utilization of emergency dental services. J Dent Sci 2020;15:564-7.

doi: $10.21037 / \mathrm{jhmhp}-20-107$

Cite this article as: Beucler N, Choucha A, Boissonneau S, Graillon T, Meyer M, Farah K, Albader F, Velly L, Fournier PE, Carron R, Fuentes S, Dufour H. How to provide continuing care during the COVID-19 pandemic: 1-month experience from the Neurosurgery Department in the French University Hospital Timone in Marseille. J Hosp Manag Health Policy 2021;5:12. 